\title{
On the equivalence of the self-dual and Maxwell-Chern-Simons models coupled to Fermions
}

\author{
M. Gomes and L. C. Malacarne* and A. J. da Silva \\ Instituto de Física, USP \\ C.P. 66318 - 05315-970, São Paulo - SP, Brazil
}

(1997)

\begin{abstract}
We study the exact equivalence between the self-dual model minimally coupled with a Dirac field and the Maxwell-Chern-Simons model with nonminimal magnetic coupling to fermions. We show that the fermion sectors of the models are equivalent only if a Thirring like interaction is included. Using functional methods we verify that, up to renormalizations, the equivalence persists at the quantum level.
\end{abstract}

PACS numbers:11.10Kk, 11.10Lm

*Permanent address: Departamento de Física, Universidade Estadual de Maringá - Av. Colombo, 5790 - 87020-900, Maringá -PR,Brazil 
$(2+1)$ dimensional models have provided insights into some basic aspects of field theory as the existence of massive gauge fields and anomalous spin and statistics. In this context, it is important to establish connections between apparently unrelated models so that unifying pictures could emerge. Examples of such endeavor are the recent tentatives of bosonization, trying to blend fermions and bosons within a coherent framework. Some approaches to bosonization rest on the fact that in $(2+1)$ dimensions there are two ways to describe a single, freely propagating, spin one, massive mode. In fact, one can use either the gauge non-invariant self-dual model $(S D)$, originally proposed by Townsend et al. [1], or the topologically massive gauge invariant Maxwell-Chern-Simons model $(M C S)$ [2]. These two descriptions are actually equivalent and this equivalence holds even if the basic fields are coupled to external sources [4]. Additional studies on the connection of these models, [3 [7] have shown that the SD is a gauge fixed version of the MCS model.

The mentioned relation between the SD and the MCS models has been used to map the massive Thirring model into a theory of interacting bosons [8]. More precisely, to leading order in the inverse fermionic mass, the use of the correspondence allows to identify the Green functions of the currents of the massive Thirring model with those of the basic vector field of th MCS model. The extension of these results to the non Abelian situation has been considered by various authors $[9]$ [13].

Although the equivalence of the SD and MCS models is well established in the free field case, it remains to understand what happens when the coupling to other dynamical fields is considered. This letter is specifically devoted to the study the equivalence when the SD and MCS are coupled to Dirac matter fields. By inspection, the source current for the SD field is found to be replaced by its rotational in the MCS vector field equation. Moreover, as we shall see, in opposition to what happens in the external source case, the classical equivalence of the corresponding Dirac's equations already demands the inclusion of an additional Thirring like interaction. At the quantum level one must be more careful because some of the interactions are not perturbatively renormalizable.

We begin our analysis by recalling that, at the field equations level, the SD model 
described by

$$
\mathcal{L}_{S D}=\frac{1}{2} m^{2} f_{\mu} f^{\mu}-\frac{m}{2} \varepsilon^{\mu \nu \rho} f_{\mu} \partial_{\nu} f_{\rho}
$$

is connected to the $M C S$ model,

$$
\mathcal{L}_{M C S}=-\frac{1}{4} F_{\mu \nu} F^{\mu \nu}+\frac{1}{4} m \varepsilon^{\mu \nu \rho} A_{\mu} F_{\nu \rho}
$$

where $F_{\mu \nu}=\partial_{\mu} A_{\nu}-\partial_{\nu} A_{\mu}$, through the identification $f^{\mu} \leftrightarrow F^{\mu}=\frac{1}{m} \varepsilon^{\mu \nu \rho} \partial_{\nu} A_{\rho}$, i. e., the basic field of the SD model corresponds to the dual of the MCS field.

Consider now a Dirac field minimally coupled to a vector field specified by the SD model, so that the new Lagrangian becomes

$$
\mathcal{L}_{S D}^{\min }=\mathcal{L}_{S D}-e f_{\mu} J^{\mu}+\bar{\psi}(i \not \partial-M) \psi
$$

where $J^{\mu}=\bar{\psi} \gamma^{\mu} \psi$ and $M$ is the fermion mass [14. From the equivalence between the SD and MCS models and noticing that the same identification of the fields, namely $f^{\mu} \leftrightarrow F^{\mu}$, up a total derivative, changes the minimal coupling into a magnetic coupling , i. e.,

$$
f_{\mu} J^{\mu} \rightarrow A_{\mu} G^{\mu}
$$

where $G^{\mu}=\frac{1}{m} \varepsilon^{\alpha \beta \mu} \partial_{\alpha} J_{\beta}$, we may infer the equivalence of the model (3) with the one specified by

$$
\mathcal{L}_{M C S}^{\text {mag }}=\mathcal{L}_{M C S}-e A_{\mu} G^{\mu}+\bar{\psi}(i \not \partial-M) \psi
$$

Indeed, a direct inspection of the equations of motion for the fields $f^{\mu}$ and $A^{\mu}$,

$$
\begin{aligned}
& -m \varepsilon^{\nu \alpha \beta} \partial_{\alpha} f_{\beta}+m^{2} f^{\nu}=e J^{\nu} \\
& -m \varepsilon^{\nu \alpha \beta} \partial_{\alpha} F_{\beta}+m^{2} F^{\nu}=e G^{\nu} .
\end{aligned}
$$

shows that the models are classically equivalents if the identification

$$
f^{\mu} \leftrightarrow F^{\mu} \Longrightarrow J^{\mu} \leftrightarrow G^{\mu}
$$


is made. This indicates that the electric interaction goes into a magnetic one. However, we still have to examine Dirac's equation. We have,

$$
(i \not \partial-M) \psi=e f_{\mu} \gamma^{\mu} \psi
$$

in the case of the SD model, and

$$
(i \not \supset-M) \psi=-\frac{e}{m} \epsilon^{\alpha \beta \rho} \partial_{\alpha} A_{\rho} \gamma_{\beta} \psi
$$

for the MCS. From (7) we can express the gauge field $A_{\mu}$ in terms of the source $G^{\mu}$. We get,

$$
A_{\alpha}(x)=e \int d y D_{R \mu \alpha}(x-y) G^{\mu}(y)
$$

where, in momentum space,

$$
D_{R \mu \alpha}(k)=\frac{1}{k^{2}-m^{2}+i \epsilon k_{0}}\left[g_{\mu \alpha}-\frac{k_{\mu} k_{\alpha}}{k^{2}+i \epsilon k_{0}}-i m \varepsilon_{\mu \alpha \rho} \frac{k^{\rho}}{k^{2}+i \epsilon k_{0}}\right]+\text { gauge fixing term }
$$

is the retarded propagator for the self dual field. Replacing (11) into (10) one obtains

$$
(i \not \supset-M) \psi=e f_{\mu} \gamma^{\mu} \psi+\frac{e^{2}}{m^{2}} J^{\beta} \gamma_{\beta} \psi
$$

where

$$
f_{\mu}(x)=e \int d y \Delta_{R \mu \alpha}(x-y) J^{\alpha}(y)
$$

and

$$
\Delta_{R \mu \alpha}(k)=\frac{1}{k^{2}-m^{2}+i \epsilon k_{0}}\left[g_{\mu \alpha}-\frac{k_{\mu} k_{\alpha}}{m^{2}}-i m \varepsilon_{\mu \alpha \rho} \frac{k^{\rho}}{m^{2}}\right]
$$

is the retarded propagator for the MCS field. Basic to this result is the algebraic identity

$$
-\frac{i}{m} \epsilon^{\alpha \beta \mu} k_{\alpha} D_{R \mu \nu}(k) \frac{i}{m} \epsilon^{\sigma \rho \nu} k_{\sigma}=\Delta_{R}^{\beta \rho}(k)+\frac{g^{\beta \rho}}{m^{2}}
$$

relating the SD and MCS propagators.

Summing up, our result shows that the fermionic sectors of the two models will agree only if one includes in one of the models a Thirring like interaction. It should be clear that 
the Thirring interaction could be either incorporated to the SD model, as we did, or to the MCS model, as we will do shortly.

We now want to investigate to what extension the equivalence (8) holds at the quantum level. Here we expect some problems as the system described by (3) is perturbatively renormalizable, whereas the Lagrangian (5) is non-renormalizable. This can be seen by direct power counting. The mass dimensions of the $A_{\mu}$ and $\psi$ are $1 / 2$ and 1 , respectively. Therefore the mass dimensions of the magnetic and of the Thirring interactions are equal to $7 / 2$ and 4, respectively,spoiling the renormalizability of the models. However this difficulty can be eventually surmounted. For example, if there are $N$ fermions the theories turns out to be $1 / N$ expandable and in this context they are renormalizable. For a solid consideration, we will employ the generating functional

$$
Z(\psi)=\int D f^{\mu} D A^{\nu} \exp \left[i \int \mathcal{L}(f, A, \psi)\right]
$$

using a master Lagrangian which generalizes (3) and (5),

$$
\mathcal{L}=\frac{m^{2}}{2} f_{\mu} f^{\mu}-m^{2} f_{\mu} F^{\mu}+\frac{m^{2}}{2} F^{\mu} A_{\mu}-e J_{\mu} f^{\mu}-e A^{\mu} g_{\mu}+\frac{\lambda}{2}\left(\partial_{\mu} A^{\mu}\right)^{2}+\mathcal{L}_{D}
$$

where $\mathcal{L}_{D}$ is the free Dirac Lagrangian and $g_{\mu}, J_{\mu}$ are matter currents depending only on the fields $\psi$ and $\bar{\psi}$. We take $J_{\mu}$ as the current $\bar{\psi} \gamma_{\mu} \psi$ but leave the specific form of $g_{\mu}$ unspecified for the time being. Integrating over the field configurations $f_{\mu}$ gives

$$
Z(\psi)=\int D A^{\mu} \exp \left[i \int \mathcal{L}_{e f f}^{(1)}(A, \psi)\right]
$$

where

$$
\begin{aligned}
\mathcal{L}_{e f f}^{(1)}(A, \psi)= & -\frac{1}{4} F_{\mu \nu} F^{\mu \nu}+\frac{1}{4} m \varepsilon^{\mu \nu \rho} A_{\mu} F_{\nu \rho}+\frac{\lambda}{2}\left(\partial_{\mu} A^{\mu}\right)^{2}- \\
& e A^{\mu}\left(g_{\mu}+G_{\mu}\right)-\frac{e^{2}}{2 m^{2}} J^{\mu} J_{\mu}+\mathcal{L}_{D} .
\end{aligned}
$$

On the other hand, integrating over $A_{\mu}$ furnishes

$$
Z(\psi)=\int D f^{\mu} \exp \left[i \int \mathcal{L}_{e f f}^{(2)}(f, \psi)\right]
$$


where

$$
\begin{aligned}
\mathcal{L}_{\text {eff }}^{(2)}(f, \psi)= & \frac{m^{2}}{2} f^{\mu} f_{\mu}-\frac{m}{2} \varepsilon_{\alpha \beta \gamma} f^{\alpha} \partial^{\beta} f^{\gamma}-e f^{\alpha}\left[J_{\alpha}+\left(g_{\alpha \beta}-\frac{\partial_{\alpha} \partial_{\beta}}{\partial^{2}}\right) g^{\beta}\right]+ \\
& \frac{e^{2}}{2 m} g_{\mu}\left[\varepsilon^{\mu \beta \nu} \frac{\partial_{\beta}}{\partial^{2}}+\frac{1}{\lambda} \frac{\partial^{\mu} \partial^{\nu}}{\left(\partial^{2}\right)^{2}}\right] g_{\nu}+\mathcal{L}_{D} .
\end{aligned}
$$

We may now consider some possibilities:

1. $g_{\mu}=0$. In this situation, (20) and (22) prove the quantum equivalence of the models previously discussed.

2. If instead we take $g_{\mu}=-G_{\mu}$ then the MCS field decouples whereas the fermions interacts through the Thirring interaction. This model of noninteracting fermions and vector fields is equivalent to the model in which, besides a self interaction, the fermions are coupled through a self dual field. This later model is described by the Lagrangian

$$
\mathcal{L}_{e f f}^{(2)}(f, \psi)=\frac{m^{2}}{2} f^{\mu} f_{\mu}-\frac{m}{2} \varepsilon_{\alpha \beta \gamma} f^{\alpha} \partial^{\beta} f^{\gamma}-e f^{\alpha}\left(J_{\alpha}-G_{\alpha}\right)-\frac{e^{2}}{2 m^{3}} \varepsilon_{\alpha \beta \gamma} J^{\alpha} \partial^{\beta} J^{\gamma}
$$

3. As a last possibility we take $g^{\mu}=-J^{\mu}$. As it happens (22) becomes

$$
\mathcal{L}_{\text {eff }}^{(2)}=\mathcal{L}_{S D}+\mathcal{L}_{D}+\frac{e^{2}}{2 m} \varepsilon^{\mu \beta \nu} J_{\mu} \frac{\partial_{\beta}}{\partial^{2}} J_{\nu}
$$

describing free self dual vector fields and self interacting fermions. By (20) this model is then equivalent to the one in which fermions and vector fields interact as specified by the effective Lagrangian

$$
\mathcal{L}_{\text {eff }}^{(1)}=\mathcal{L}_{M C S}+\mathcal{L}_{D}+e A^{\mu}\left(J_{\mu}-G_{\mu}\right)-\frac{e^{2}}{2 m^{2}} J_{\mu} J^{\mu}+\frac{\lambda}{2}\left(\partial_{\mu} A^{\mu}\right)^{2}
$$

It should be clear that the equivalence of models that we just proved is very particular, being a direct consequence of an algebraic identity. For example it does not seem to hold in the scalar case. Indeed, in that case the gauge current depends explicitly on the gauge field and, accordingly, the functional determinant has a non trivial dependence on the matter fields.

The models considered involve perturbatively non renormalizable couplings, namely the Thirring and the magnetic interactions. However, if the fermion field is an $N$ component 
field the models are $1 / N$ expandable and in this context they are renormalizable. For the Thirring model this was proved in [15]. Here we examine the case when the MCS field interacts through the magnetic coupling, $A_{\mu} G^{\mu}$. It is easy to verify that the propagator for the vector field behaves as the momentum $p$ tends to infinity as $1 /\left(p^{2}\right)^{3 / 2}$. Power counting, therefore, establishes that the degree of superficial divergence of a generic graph $G$ is

$$
d(G)=3-N_{F}
$$

where $N_{F}$ is the number of external fermion lines of $G$. Apparently, here we run into a difficulty since $d(G)$ does not decreases as the number of external vector lines increases. However, (26) does not take into account that, for an external vector line, the momentum factor associated with the vertex to which the line is attached does not depend on the integration variables so that the effective degree of divergence is lowered to

$$
3-N_{F}-N_{B}
$$

which, as expected, is the same as in the Thirring model. It is also easy to verify that the counterterms needed to render finite the Green functions have the same form of terms already present in the original Lagrangian. This means that the theory is renormalizable as, stated.

\section{ACKNOWLEDGMENTS}

This work was partially supported by Conselho Nacional de Desenvolvimento Científico e Tecnológico (CNPq) and Coordenação de Aperfeiçoamento de Pessoal de Nível Superior (Capes). 


\section{REFERENCES}

[1] P. K. Townsend, K. Pilch and P. Van Nieuwenhuizen, Phys. Lett. B136 (1984) 38.

[2] S. Deser, R. Jackiw, Annals Phys. 140 (1982) 372.

[3] R. Gianvittorio, A. Restuccia and J. Stephany, Mod. Phys. Lett. A6 (1991) 2121.

[4] S. Deser, R. Jackiw, Phys. Lett. B139 (1984) 371.

[5] R. Banerjee, H. J. Rothe and K. D. Rothe, Phys. Rev. D52 (1995) 3750.

[6] I. A. Batalin and E. S. Fradkin, Nucl. Phys. B279 (1987) 514.

[7] R. Banerjee, H. J. Rothe and K. D. Rothe, Phys. Rev D55 (1997) 6339.

[8] C. Fradkin, F. A. Schaposnik, Phys. Lett. B338 (1994) 253; K. Kondo, Prog. Theor. Phys. 94 (1995) 869.

[9] A. Karlhede et al, Phys. Lett. B186 (1987) 96.

[10] P. J. Arias, A. Restuccia, Phys. Lett B347 (1993) 1241; P. J. Arias, L. Leal and A. Restuccia, Phys. Lett B 367 ( 1996) 170.

[11] N. Bralić, E. Fradkin, V. Manias and F. A. Schaposnik, Nucl. Phys. B 446 (1995) 144.

[12] J. C. Le Guillou, E. Moreno, C. Núnez and F. A. Schaposnik, Nucl. Phys. B 484 (1997) 682.

[13] N. Banerjee, R. Banerjee, S. Ghosh, Nucl. Phys. B 481 (1996) 421.

[14] We use natural units $(c=\hbar=1)$ and our metric is $g_{00}=-g_{11}=-g_{22}=1$. The fully antsymetric tensor $\varepsilon^{\mu \nu \rho}$ is normalized such that $\varepsilon^{012}=1$. For the $\gamma$-matrices we adopt the representation $\gamma^{0}=\sigma^{3}, \gamma^{1}=\sigma^{1}, \gamma^{2}=i \sigma^{2}$, where $\sigma^{i}, i=1,2,3$, are the Pauli spin matrices.

[15] M. Gomes, R. S. Mendes, R. F. Ribeiro and A. J. da Silva, Phys. Rev. D43, (1991) 3516. 